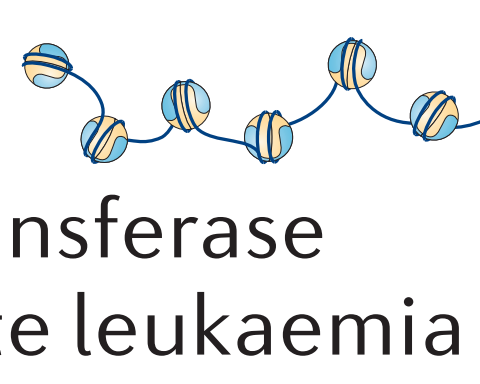

\title{
Histone methyltransferase mutations promote leukaemia
}

Recent sequencing studies of human cancers have identified tumourspecific mutations in genes encoding proteins that function in chromatin regulation, although their functional importance has not always been clear. A study by Zhu et al. has identified mutations in the histonelysine $N$-methyltransferase SETD2 and has shown that these mutations cooperate with other genetic aberrations in leukaemia.

Leukaemias frequently contain chromosomal rearrangements, but these changes are not by themselves leukaemogenic. To identify mutations that cooperate with primary chromosomal translocations, the authors used whole-genome sequencing on monozygotic twins who were discordant for mixed lineage leukaemia gene $(M L L)$-associated acute myeloid leukaemia. They identified an MLL-NRIP3 (nuclear receptor interacting protein 3) fusion gene, as well as mutations in SETD2,

this knowledge could provide alternative treatments for leukaemias and potentially other cancers that harbour

SETD2 mutations

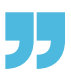

which encodes the only histonemodifying enzyme that can catalyse trimethylation (me3) of histone 3 , lysine 36 (H3K36).

They focused on these SETD2 mutations and carried out Sanger sequencing on an additional 241 patients with acute myeloid or lymphoblastic leukaemia. $6.2 \%$ of these patients had SETD2 mutations. Of these, the patients who had both alleles of SETD2 mutated had two distinct mutations, which indicates that SETD2 mutations are biallelic.
Interestingly, $22.2 \%$

of patients with $M L L$ rearrangements also had SETD2 mutations, and most patients with SETD2 mutations (86.7\%) also had one other genetic aberration.

The majority of mutations in SETD2 resulted in truncation of the protein - specifically, the loss of the carboxy-terminal SRI (SET2RPB1 interacting) domain, which is required for the recruitment of SETD2 to its target loci. Thus, the loss of the SRI domain results in the loss of SETD2 function. This was manifested as a global decrease in $\mathrm{H} 3 \mathrm{~K} 36 \mathrm{me} 3$ levels in leukaemias from patients with SETD2 mutations relative to H3K36me3 levels in patients with leukaemias with wild-type SETD2.

Using several different mouse models of pre-leukaemic diseases, the authors then asked whether SETD2 mutations alone are sufficient to initiate leukaemia. Colony-forming assays with haematopoietic stem and progenitor cells transfected with Setd 2 short hairpin RNAs showed that Setd 2 knockdown did not significantly affect the growth of wild-type cells, whereas it increased the yield of pre-leukaemic cell colonies. This suggests that SETD2 loss is important in the maintenance and progression of leukaemia but is not sufficient to initiate it.

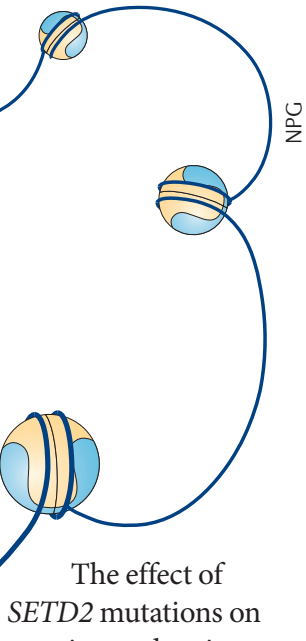

the progression and maintenance of leukaemia was determined by knocking down $\operatorname{Set} 2$ in primary leukaemic cells from leukaemic mice and serially transplanting them into sublethally irradiated recipients. Setd 2 knockdown caused a higher frequency of leukaemia-initiating cells and leukaemia cells, as well as a shorter latency and a more severe phenotype in the transplanted mice. Finally, the authors used mRNA sequencing to show that Setd2 knockdown in these cells led to an increase in the expression of genes that are part of the human leukaemia stem cell signature and the human embryonic stem cell signature.

These findings indicate that SETD2 might function as a tumour suppressor in acute leukaemia, and this knowledge could provide alternative treatments for leukaemias and potentially other cancers that harbour SETD2 mutations. It remains unclear how the loss of SETD2 cooperates with other mutations, such as $M L L$ translocations, and why it leads to a stem cell-like gene signature.

Isabel Lokody

ORIGINAL RESEARCH PAPER Zhu, X. et al. Identification of functional cooperative mutations of SETD2 in human acute leukemia. Nature Genet. http://dx.doi.org/10.1038/ng.2894 (2014) 